\title{
Outcomes of Trabeculectomy at a Newly Established Glaucoma Clinic in Khyber Pakhtunkhwa
}

\author{
Ali Hassan Nasir ${ }^{1}$, Mashal Bano ${ }^{2}$, Yousaf Jamal Mahsood ${ }^{3}$ \\ ${ }^{1-3}$ Hayatabad Medical Complex, Peshawar
}

\begin{abstract}
Purpose: To determine the outcomes of trabeculectomy in patients with open angle glaucoma at a newly established glaucoma clinic in a tertiary care hospital of Khyber Pakhtunkhwa.
\end{abstract}

Study Design: Retrospective chart review.

Place and Duration of Study: Glaucoma Clinic, Hayatabad Medical Complex, Peshawar from May 2018 to December 2019.

Methods: A retrospective chart review of patients of 18 years age or above and who underwent trabeculectomy for open angle glaucoma, with at least one follow-up visit were included. Data was collected for age, gender, type of glaucoma, preoperative best-corrected visual acuity (BCVA), intraocular pressure (IOP), number of preoperative/post-operative topical anti-glaucoma medications, indication for trabeculectomy, preoperative comorbidities and postoperative complications. Data were analyzed using SPSS version 23. The student's paired t-test was used for comparative analysis of the same group. A p-value of $<0.05$ was considered significant.

Results: A total of 20 eyes of 20 patients were included in this study, 13 (65\%) patients were males, and the mean age was $54.60 \pm 18.22$ years. There was a statistically significant change in mean IOP postoperatively (35.60 \pm 13.28 versus $11.2 \pm 6.1, P<0.001)$ and number of antiglaucoma medication $(2.95 \pm 1.19$ versus $0.4 \pm$ $0.99, P<0.001)$. While there was no significant change in best-corrected visual acuity $(1.35 \pm 0.88$ versus $1.06 \pm$ $0.72, p=0.36)$.

Conclusion: Trabeculectomy performed at the newly established glaucoma clinic showed a significant reduction in Intraocular pressure without compromising the visual acuity of the study participants.

Key Words: Trabeculectomy; Open Angle Glaucoma; Intraocular pressure.

How to Cite this Article: Nasir AH, Bano M, Mahsood YJ. Outcomes of Trabeculectomy at a Newly Established Glaucoma Clinic in Khyber Pakhtunkhwa. Pak J Ophthalmol. 2022, 38 (1): 48-51.

Doi: $10.36351 /$ pjo.v38i1.1306

Correspondence: Yousaf Jamal Mahsood

Department of Ophthalmology, Hayatabad Medical

Complex, Peshawar

Email: yousaf82@hotmail.com

Received: June 21, 2021

Accepted: November 30, 2021

\section{INTRODUCTION}

Glaucoma is one of the leading causes of irreversible blindness with 76 million people affected by this disease globally. ${ }^{1}$ However, less than half of the population suffering from glaucoma are aware of their disease. ${ }^{2}$ This is the main reason that many glaucoma patients present when the disease is in an advanced stage. ${ }^{3}$ Pakistan has a burden of over 1.8 million people suffering from glaucoma with more and more people becoming blind to this disease due to delayed presentation primarily due to lack of knowledge. ${ }^{4}$

Control of intraocular pressure (IOP) can be achieved with IOP-lowering topical medications, 
lasers, or surgery. Trabeculectomy, a filtration surgery, first introduced by Cairns in 1968 and then modified subsequently by Watson in 1970, has been the Gold Standard surgical procedure for Glaucoma. ${ }^{5}$ Studies have shown that progression of optic neuropathy is controlled to some extent in patients undergoing trabeculectomy over 3-6 years. ${ }^{6}$ Trabeculectomy, although a successful procedure, has variable outcomes due to age, gender, type of glaucoma, and ethnicity. $^{7}$ Although data is available on the indications, technique, and success of Trabeculectomy ${ }^{8}$, yet we have no national consensus.

Glaucoma is emerging as a new subspecialty in Pakistan and our hospital has recently started regular glaucoma clinic. The main purpose of this study is to determine the outcomes of trabeculectomy in patients presenting to this newly established glaucoma clinic in a tertiary care hospital in Khyber Pakhtunkhwa.

\section{METHODS}

A retrospective chart review was conducted in the Glaucoma Clinic of Ophthalmology Department of Hayatabad Medical Complex, Peshawar. This study was conducted after approval of the hospital ethical committee (Ref. No. 376/HEC/B\&PSC/2020) and followed the Helsinki declaration guidelines. Charts of those patients who underwent trabeculectomy between May 2018 and December 2019 were searched. Patients with open angle glaucoma who were 18 years or above at the time of trabeculectomy and with at least one follow-up visit were eligible for inclusion in the study. Data was collected for age, gender, type of glaucoma, preoperative best-corrected visual acuity (BCVA), intraocular pressure (IOP), number of preoperative topical antiglaucoma medications, indication for trabeculectomy, and any preoperative comorbidities. The follow-up data was BCVA, IOP, number of antiglaucoma medications, postoperative complications like loss of visual acuity, intraocular pressure, shallow anterior chamber, choroidal detachment, hypotony, maculopathy, macular edema, and disc swelling.

The technique used in all surgeries was the same and details were retrieved from surgical records of the patients. All surgeries were fornix based done under peri-bulbar anesthesia. Corneal traction suture with 7/0 vicryl was used to expose the superior surface of the conjunctiva. A $4 \mathrm{~mm}$ conjunctival flap measured with calipers was made with Westcott scissors, wet-field cautery was used to clear the scleral vessels, and $0.4 \mathrm{mg} / \mathrm{ml}$ Mitomycin C soaked cotton sponges were placed on the sclera for 2 minutes, which was then thoroughly washed with balanced saline solution. A $3 \times 3 \mathrm{~mm}$ partial-thickness triangular scleral flap was made and trabeculectomy was performed using kelly's punch and iridectomy was performed with scissors. The flap was secured with 3 slip knots using 10/0 nylon thread. A side port was made to check the patency of the bleb and the anterior chamber was maintained. Conjunctiva was sutured with two 10/0 nylon wing sutures. Subconjunctival dexamethasone $4 \mathrm{mg} / \mathrm{ml}$ was injected in the inferior fornix at the end of the surgery. After the surgery, all patients were prescribed topical Moxifloxacin eye drops four times a day, topical dexamethasone eye drops two hourly, topical tobramycin and dexamethasone eye ointment thrice daily. Data were analyzed using SPSS version 23 (IBM Corp., Armonk, N.Y., USA). For categorical variables (like laterality, diagnosis, indications, and complications), frequencies with valid percentages were calculated. For continuous variables (like age, IOP, visual acuity, and the number of antiglaucoma medications), mean \pm standard deviation were calculated. The student's paired T-test was used for comparative analysis of the same group. A p-value of $<0.05$ was considered significant.

\section{RESULTS}

A total of 54 patients underwent trabeculectomy from May 2018 till December 2019. Out of these, 33 patients underwent trabeculectomy for Open Angle Glaucoma while 21 patients underwent trabeculectomy for causes other than Open Angle Glaucoma like Angle Closure Glaucoma and congenital Glaucoma. Out of the 33 patients, records of 10 patients could not be traced while 3 patients did not have a post-operative visit and were excluded from the study. Baseline demographics are shown in Table 1.

Comparison of preoperative and postoperative results are shown in Table 2. A statistically significant reduction in both IOP and antiglaucoma medication was noted $(\mathrm{P}<0.001)$. There was no significant statistical change in final BCVA as compared to the preoperative value $(\mathrm{P} \leq 0.364)$. Table 3 highlights prior ocular comorbidities, 7 patients $(35 \%)$ had previous cataract surgery and 3 patients $(15 \%)$ had a prior trabeculectomy done. No patient had any intraoperative complications. 
Table 1: Baseline Demographics of Our Study Population.

\begin{tabular}{|c|c|c|c|}
\hline \multicolumn{2}{|c|}{ Characteristics } & \multirow{2}{*}{$\begin{array}{l}\begin{array}{l}\text { Frequency } \\
(\boldsymbol{\%}) \mathbf{N}=\mathbf{2 0}\end{array} \\
13(65)\end{array}$} & \multirow{11}{*}{ Mean (SD) } \\
\hline \multirow{2}{*}{ Gender } & Male & & \\
\hline & Female & $7(35)$ & \\
\hline \multirow{2}{*}{$\begin{array}{l}\text { Laterality } \\
\text { of the eye }\end{array}$} & Right & $5(25)$ & \\
\hline & Left & $15(75)$ & \\
\hline \multirow{2}{*}{ Diagnosis } & POAG & $13(65)$ & \\
\hline & SOAG & $7(35)$ & \\
\hline \multirow{4}{*}{ Indications } & Uncontrolled IOP & $14(70)$ & \\
\hline & $\begin{array}{l}\text { Progression despite } \\
\text { treatment }\end{array}$ & $2(10)$ & \\
\hline & Poor compliance & $2(10)$ & \\
\hline & Others & $2(10)$ & \\
\hline \multicolumn{3}{|c|}{ Age (Years) } & $54.6 \pm 18.22$ \\
\hline \multirow{3}{*}{\multicolumn{3}{|c|}{$\begin{array}{l}\text { Pre-Operative Anti Glaucoma Medications } \\
\text { Best Corrected Visual Acuity (LogMAR units) } \\
\text { Pre-Operative Intraocular Pressure (mmHg) }\end{array}$}} & $2.95 \pm 1.19$ \\
\hline & & & $1.35 \pm 0.88$ \\
\hline & & & $35.60 \pm 13.28$ \\
\hline
\end{tabular}

$\mathrm{N}=$ Total number of eyes, $\%=$ Percentage, $\mathrm{POAG}=$ Primary Open Angle Glaucoma, SOAG = Secondary Open Angle Glaucoma, IOP = Intraocular Pressure, SD = Standard deviation, $\operatorname{LogMAR}=\log$ of minimum angle of resolution, $\mathrm{mmHg}=$ Millimeter of mercury.

Table 2: Comparison of Preoperative and Postoperative Results.

\begin{tabular}{lccc}
\hline Characteristics & $\begin{array}{c}\text { Preoperative } \\
\text { (SD) }\end{array}$ & $\begin{array}{c}\text { Final Visit } \\
\text { (SD) }\end{array}$ & $\begin{array}{c}\text { p- } \\
\text { value* }\end{array}$ \\
\hline BCVA (LogMAR & $1.35(0.88)$ & $1.06(0.72)$ & 0.36 \\
units) & $35.60(13.28)$ & $11.2(6.1)$ & $<0.001$ \\
IOP (mmHg) & $2.95(1.19)$ & $0.4(0.99)$ & $<0.001$ \\
No. of antiglaucoma & medications & &
\end{tabular}

IOP = Intraocular Pressure, $\mathrm{SD}=$ Standard deviation, LogMAR = Log of minimum angle of resolution, $\mathrm{mmHg}=$ Millimeter of mercury, No. $=$ Number. ${ }^{*}$ paired t-test was applied.

Table 3: Ocular Comorbidities.

\begin{tabular}{lcc}
\hline Characteristics & Frequency & Percentage \\
\hline No Ocular Comorbidity & 8 & 40 \\
Cataract surgery & 7 & 35 \\
Uveitis & 1 & 5 \\
Trabeculectomy & 3 & 15 \\
Others & 1 & 5 \\
Total & 20 & 100 \\
\hline
\end{tabular}

\section{DISCUSSION}

The purpose of this study was to determine the outcomes of trabeculectomy in patients performed at a newly established glaucoma clinic. Our Study showed that trabeculectomy, has a significant impact on the reduction of IOP and medication along with stabilization of visual acuity. ${ }^{8,9}$
The most common indication for trabeculectomy in our study was uncontrolled IOP $(70 \%)$, while the progression of Optic nerve head changes and deterioration of visual field defects was noted in a study conducted at Manchester Royal Eye Hospital. ${ }^{10}$ The reason for this difference is that we have reported short-term results as our glaucoma clinic is newly established. On the other hand, there was no statistically significant change in visual acuity of our patients which is in line with other studies reported. ${ }^{4,9,11}$

The mean decrease in IOP noted in our study was similar to the results of a study conducted by Shahid et al. ${ }^{4}$ Similarly, a study conducted in Nigeria also reported a significant reduction in IOP. ${ }^{12}$ Even though a higher IOP reduction was reported in our study, setting a target pressure is always individualized according to the patient individual characteristics. ${ }^{9}$ The goal of treatment with control of IOP being to help stabilize progressive visual field loss. ${ }^{13} \mathrm{We}$ also found a significant reduction in antiglaucoma medications in our patients which was similar to a study conducted in Brazil. ${ }^{11}$ Similar drop in the use of antiglaucoma medications was reported in a study conducted in Sweden. ${ }^{14}$

New surgical technologies for example shunts, canaloplasty, Trabectome and endoscopic cyclophotocoagulation (ECP), have been developed to provide safe and effective control of intraocular pressure but conventional trabeculectomy has an important role in glaucoma management. ${ }^{15}$

With the establishment of a new glaucoma clinic comes its own set of difficulties in adopting a procedure and treatment guidelines that are according to the population being treated. Outcomes of treatment are usually masked when data is not taken into account to highlight the potential benefits and outline the shortcomings to comply with international standards. Comparing the results achieved in our study, it is safe to say that trabeculectomy, as a procedure is having the desired effect as mentioned in different published studies.

This study, being retrospective in nature was limited by its design along with the lack of availability of all the files with the proper data recording. The average follow-up was limited to only one follow-up visit. We recommend a multi-centered study with longer follow-up, which will further contribute to the data. 


\section{CONCLUSION}

Trabeculectomy performed at the newly established glaucoma clinic showed good results in controlling IOP along with reducing antiglaucoma medication.

\section{Ethical Approval}

The study was approved by the Institutional review board/ Ethical review board.

(376/HEC/B\&PSC/2020).

\section{Conflict of Interest}

Authors declared no conflict of interest.

\section{REFERENCES}

1. Tham Y-C, Li X, Wong TY, Quigley HA, Aung T, Cheng C-Y. Global Prevalence of Glaucoma and Projections of Glaucoma Burden through 2040 A Systematic Review and Meta-Analysis. Ophthalmology, 2014; 121: 2081-2090.

2. Quigley HA. Number of people with glaucoma worldwide. Br J Ophthalmol. 1996; 80 (5): 389-393.

3. Thapa SS, Paudyal I, Khanal S, Twyana SN, Paudyal G, Gurung R, et al. A population-based survey of the prevalence and types of glaucoma in Nepal: The Bhaktapur glaucoma study. Ophthalmology, 2012 Apr; 119 (4): 759-764.

4. Shahid E, Fasih U, Shaikh A. Outcome of Conventional Trabeculectomy in Terms of Intraocular Pressure and Visual Acuity in Primary Open Angle Glaucoma. Pakistan J Ophthalmol. 2020; 36 (4): 386390.

5. Koike KJ, Chang PT. Trabeculectomy: A Brief History and Review of Current Trends. Int Ophthalmol Clin. 2018; 58 (3): 117-133.

6. Nouri-Mahdavi $\mathbf{K}$, Brigatti $\mathbf{L}$, Weitzman $\mathbf{M}$, Caprioli J. Outcomes of Trabeculectomy for Primary Open-angle Glaucoma. Ophthalmology, 1995; 102 (12): 1760-1769.

7. Hah MH, Omar RNR, Jalaluddin J, Jalil NFA, Selvathurai A. Outcome of trabeculectomy in hospital Melaka, Malaysia. Int J Ophthalmol. 2012; 5 (3): 384388.

8. Gedde SJ, Feuer WJ, Lim KS, Barton K, Goyal S, Ahmed IIK, et al. Treatment Outcomes in the Primary Tube Versus Trabeculectomy Study after 3 Years of Follow-up. In: Ophthalmology. Elsevier Inc.; 2020: p. 333-345.
9. Tabassum G, Ghayoor I, Pak RA. The Effectiveness of Conventional Trabeculectomy in Controlling Intraocular Pressure in Our Population. Pak J Ophthalmol. 2013; 29 (1): 26-30.

10. Shiwani HA, Naqvi S, Cristian C, Au L, Spencer $\mathrm{AF}$, Fenerty $\mathbf{C H}$, et al. Outcomes of Primary Trabeculectomy from Two Same-Centre Cohorts Ten Years Apart. Journal of Glaucoma, 2021 May 27. Doi: 10.1097/IJG.0000000000001887. Epub ahead of print. PMID: 34049346.

11. Abe RY, Shigueoka LS, Vasconcellos JPC, Costa VP. Primary Trabeculectomy Outcomes by Glaucoma Fellows in a Tertiary Hospital in Brazil. J Glaucoma, 2017; 26 (11): 1019-1024.

12. Adegbehingbe B., Majemgbasan T. A review of trabeculectomies at a Nigerian teaching Hospital. Ghana Med J. 2010 Jun. 7; 41 (4).

13. Rao HL, Addepalli UK, Jonnadula GB, Kumbar T, Senthil S, Garudadri CS. Relationship between intraocular pressure and rate of visual field progression in treated glaucoma. J Glaucoma, 2013 Dec; 22 (9): 719-724.

14. Binibrahim IH, Bergström AK. The role of trabeculectomy in enhancing glaucoma patient's quality of life. Oman J Ophthalmol. 2017; 10 (3): 150-154.

15. Brooks AM, Gillies WE. New techniques in glaucoma surgery. Br J Ophthalmol. 2000; 84 (12):1339-41.

Doi: $10.1136 /$ bjo.84.12.1339.

PMID: 11090469; PMCID: PMC1723352.

\section{Authors' Designation and Contribution}

Ali Hassan Nasir; Trainee Medical Officer: Design, Literature search, Data acquisition, Statistical analysis, Manuscript preparation, Manuscript editing, Manuscript review.

Mashal Bano; Trainee Medical Officer: Design, Literature search, Data acquisition, Statistical analysis, Manuscript preparation, Manuscript editing, Manuscript review.

Yousaf Jamal Mahsood; Assistant Professor: Concept, Design, Literature search, Data acquisition, Data analysis, Statistical analysis, Manuscript preparation, Manuscript editing, Manuscript review. 